\title{
THE OLD ENVIRONMENT OF THE FAINT CALCIUM-RICH SUPERNOVA SN 2005cz
}

\author{
Hagai B. Perets ${ }^{1}$, Avishay Gal-yam ${ }^{2}$, R. Mark Crockett ${ }^{3}$, Joseph P. Anderson ${ }^{4}$, Phil A. James ${ }^{5}$, Mark Sullivan ${ }^{3}$, \\ James D. NeILl ${ }^{6}$, AND Douglas C. LeONARD ${ }^{7}$ \\ ${ }^{1}$ Harvard-Smithsonian Center for Astrophysics, 60 Garden Street, Cambridge, MA 02338, USA \\ ${ }^{2}$ Weizmann Institute of Science, POB 26, Rehovot, Israel \\ ${ }^{3}$ Department of Physics, University of Oxford, Denys Wilkinson Building, Keble Road, Oxford OX1 3RH, UK \\ ${ }^{4}$ Departamento de Astronomia, Universidad de Chile, Casilla 36-D, Santiago, Chile \\ ${ }^{5}$ Astrophysics Research Institute, Liverpool John Moores University, Twelve Quays House, Egerton Wharf, Birkenhead CH41 1LD, UK \\ ${ }^{6}$ California Institute of Technology, 1200 East California Boulevard, Pasadena, CA 91125, USA \\ ${ }^{7}$ Department of Astronomy, San Diego State University, San Diego, CA 92182, USA \\ Received 2010 December 1; accepted 2011 January 18; published 2011 January 31
}

\begin{abstract}
The supernova SN $2005 \mathrm{cz}$ has recently attracted some attention due to the fact that it was spectroscopically similar to type $\mathrm{Ib}$ supernovae ( $\mathrm{SNe} \mathrm{Ib}$ ), a class that is presumed to result from the core collapse of massive stars, yet it occurred in an elliptical galaxy, where one expects very few massive stars to exist. Two explanations for this remarkable event were put forward. Perets et al. associate SN 2005cz with the class of Ca-rich, faint SNe Ib, which likely result from old double-white-dwarf systems with an He-rich secondary. On the other hand, Kawabata et al. suggest that SN $2005 \mathrm{cz}$ is indeed a core-collapse event (in a binary system), albeit of a star at the lower end of the mass range, $10-12 M_{\odot}$. The existence of this star in its elliptical host is explained as resulting from low-level star formation (SF) activity in that galaxy. Here we present extensive observations of the location of SN 2005cz, sensitive to a variety of SF tracers, including optical spectroscopy, $\mathrm{H} \alpha$ emission, UV emission, and Hubble Space Telescope photometry. We show that NGC 4589, the host galaxy of SN 2005cz, does not show any signatures of a young stellar population or recent SF activity either close to or far from the location of SN 2005cz.
\end{abstract}

Key words: galaxies: star formation - H II regions - stars: formation - supernovae: general - supernovae: individual (SN 2005cz) - white dwarfs

Online-only material: color figures

\section{INTRODUCTION}

Recently, a novel type of faint supernovae ( $\mathrm{SNe}$ ) with peculiar properties has been discovered. A group of eight such events has been identified, all spectroscopically similar to type $\mathrm{Ib} \mathrm{SNe}$ (SNe Ib), but faint (typical absolute peak magnitude of $\sim-15$ ) and calcium-rich (Perets et al. 2010). Although SNe Ib are generally thought to result from the core collapse of massive stars (e.g., Filippenko 1997), a large fraction of the host galaxies of these faint, Ca-rich SNe are early-type galaxies. Additionally, the ejecta mass of $\mathrm{SNe}$ in this subclass appear to be very low (e.g., $\sim 0.3 M_{\odot}$ found for SN $2005 \mathrm{E}$, Perets et al. $2010 ; \lesssim 1 M_{\odot}$ found for SN 2005cz, Kawabata et al. 2010), less than expected and observed for core-collapse $\mathrm{SNe}$ of any type. These $\mathrm{SNe}$ were therefore suggested to originate from a different process involving the thermonuclear explosion of a helium shell on a white dwarf (Perets et al. 2010; Shen et al. 2010; Waldman et al. 2010). Nevertheless, an alternative scenario involving a core collapse of a 10-12 $M_{\odot}$ star, which is a part of a binary, was suggested by Kawabata et al. (2010) for the origin of one of the Ca-rich SNe: SN 2005cz. Here we study this possibility and look for any evidence for star formation (SF) or a young stellar population near the location of SN 2005cz. In the following, we discuss our results from observations of various SF tracers including $\mathrm{H} \alpha$ emission, Hubble Space Telescope (HST) photometry, host galaxy spectra, and UV emission. In addition, we shortly discuss the SF and merger history of the host galaxy.

\section{STAR FORMATION TRACES}

Massive stars are usually formed and observed in giant molecular clouds and young stellar clusters or associations (Chu
\& Gruendl 2008; Schilbach \& Röser 2008). Core-collapse SNe from massive stars are therefore expected to be found close to star-forming regions (SFRs), and overall in SF galaxies. We therefore searched for SF tracers in the host galaxy both near and far from the location of SN $2005 \mathrm{cz}$.

SFRs produce two classes of emission: continuum emission from young stars and emission lines (dominated by $\mathrm{H} \alpha$ ) produced by ionized gas. We have searched for both classes of emission and obtained upper limits on the SF rates. In addition, we used HST images to look for nearby point sources that may correspond to young stars close to the SN location.

\subsection{R-band and $\mathrm{H \alpha}$ Imaging}

SN 2005cz was discovered on 2005 July 17, in the elliptical galaxy NGC 4589 (Dimai et al. 2005; R.A. 12:37:27.85, decl. 74:11:24.5). Published $\mathrm{H} \alpha$ narrowband imaging data exist for this galaxy (Goudfrooij et al. 1994). These data do not suggest any SF activity close to the location of SN $2005 \mathrm{cz}$, although Goudfrooij et al. do find a significant $\mathrm{H} \alpha+\left[\mathrm{N}_{\text {II }}\right]$ emission from the central regions of the galaxy, aligned along the minor axis where a dust lane is observed (see Figure 1 and their Figure 22).

We obtained an additional $\mathrm{H} \alpha$ image of NGC 4589 with the Liverpool Telescope (LT), and then analyzed it using similar methods to those described in detail elsewhere (Anderson \& James 2009). We detected the dust lane mentioned above as a perturbation to the isophotes in our LT $R$-band image. Note that according to Goudfrooij et al. (1994) the $\mathrm{H} \alpha+[\mathrm{N}$ II] emission is associated with the LINER nucleus, and SF is not discussed in that context; this is further supported by Ho et al. (1997) and our analysis of the galaxy spectrum (see below and in Figure 2). Moreover, the emission runs from the galactic nucleus along an axis offset to that containing the SN $2005 \mathrm{cz}$ position. Therefore, 


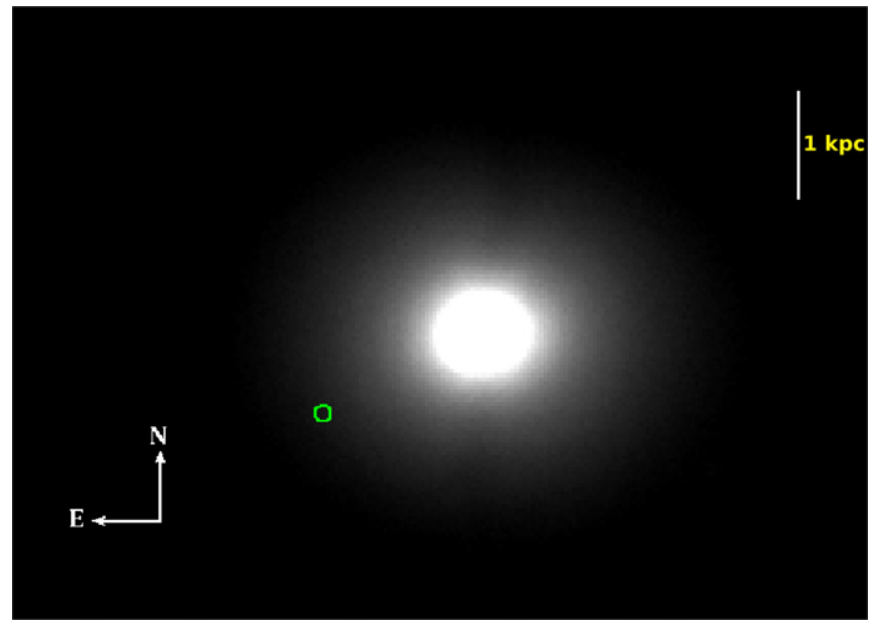

Figure 1. $R$-band imaging of SN $2005 \mathrm{cz}$ host galaxy NGC 4589. The dust lane reported by Goudfrooij et al. (1994) is weakly seen here as well far away from the reported location of the SN (marked with a circle).

(A color version of this figure is available in the online journal.)

there is no evidence in either the Goudfrooij et al. (1994) data or in ours for any line emission near the SN location and even to large distances from it ( $>1.5 \mathrm{kpc}$, see also Figure 2).

Although core-collapse $\mathrm{SNe}$ do not locally trace the $\mathrm{H} \alpha$ emission from SF regions, they do trace larger scale SF $\mathrm{H} \alpha$ emission in the host galaxies of core-collapse SNe (Anderson \& James 2009). In particular, none of the $>100 \mathrm{SNe}$ in the Anderson \& James (2009) sample were found in elliptical galaxies, and only $<3 \%$ of all core-collapse $\mathrm{SNe}$ in their survey show $\mathrm{H} \alpha$ emission at similar or larger distances from the SNe location as found for SN $2005 \mathrm{cz}$. We note that in the latter cases the emission was clearly related to $\mathrm{SF}$, whereas the $\mathrm{H} \alpha$ emission we find in the host galaxy of SN 2005cz (but far from it) is likely related to the LINER nucleus, rather than to any SF activity (see the following section).

We also used our data to determine a $3 \sigma$ upper limit for the $\mathrm{H} \alpha$ flux from the variation of the sky background, using a $2^{\prime \prime}$ aperture, of $1.3 \times 10^{-14} \mathrm{erg} \mathrm{cm}^{-2}$ (determined using a host galaxy $R$-band magnitude taken from Sandage \& Visvanathan 1978). At the distance of NGC 4589 (29.42 Mpc, taken from $\mathrm{NED}^{8}$ ), this corresponds to an $\mathrm{H} \alpha$ luminosity of $1.33 \times 10^{39} \mathrm{erg} \mathrm{s}^{-1}$. Correcting this for Galactic extinction (0.075) and the contribution from N II lines we get an $\mathrm{H} \alpha$ flux of $1.07 \times 10^{39} \mathrm{erg} \mathrm{s}^{-1} \mathrm{~cm}^{-2}$. Using the calibration from Kennicutt (1998, Equation (2)) we find an upper limit on the SF rate of $\mathrm{SFR}_{\text {limit }}=8.45 \times 10^{-3} \mathrm{M}_{\odot} \mathrm{yr}^{-1}$.

\subsection{Host Galaxy Spectrum}

We observed the location of SN $2005 \mathrm{cz}$ on 2005 July 28 using the LRIS spectrograph mounted on the Keck $10 \mathrm{~m}$ telescope on 2005 July 28 and obtained data from a 500 s exposure. The deep, two-dimensional spectral image shows no emission features within at least $1 \mathrm{kpc}$ of the location of SN $2005 \mathrm{cz}$ (see Figure 2). While emission lines from the bright galaxy nucleus are seen on the spectral image, they are $\sim 12^{\prime \prime} .1(\sim 1.7 \mathrm{kpc})$ away, and are not those of typical HiI regions (e.g., they include strong [S II] $6722 / 6734)$; rather, they present line ratios characteristic of a LINER galaxy nucleus, consistent with the LINER classification of this galaxy given by Ho et al. (1997). Thus, while $\mathrm{H} \alpha$ emission

\footnotetext{
8 http://nedwww.ipac.caltech.edu/
}

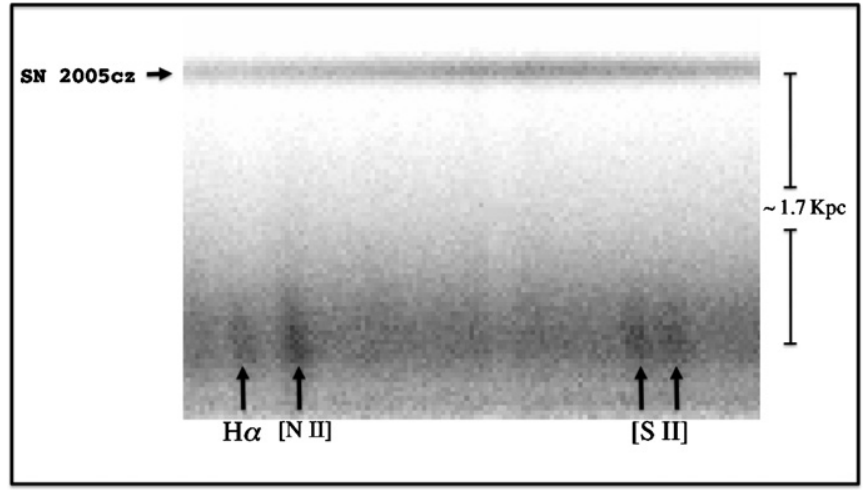

Figure 2. Close-up of the two-dimensional spectrum of SN $2005 \mathrm{cz}$, obtained in a $500 \mathrm{~s}$ exposure at the $10 \mathrm{~m}$ Keck I telescope on 2005 July 28. The slit was oriented at a position angle of $100^{\circ}$ east of north, centered on SN $2005 \mathrm{cz}$. Only the lower portion of the spectral image, which included the portion near to the galaxy's nucleus, is displayed here; the upper portion showed no emission-line activity. Arrows indicate the locations on the CCD chip of SN 2005cz (horizontal line), as well as the $\mathrm{H} \alpha$, [N II] $\lambda 6583$, and [S II] $\lambda \lambda 6722 / 6734$ emission lines from the galaxy nucleus. The relative strengths of the emission lines are consistent with the LINER classification given by Ho et al. (1997) for the nucleus of NGC 4589, and the lack of perceptible $\mathrm{H} \alpha$ emission close to the location of SN $2005 \mathrm{cz}$ in this deep spectral image is consistent with the absence of any other indicator of ongoing SF activity in the galaxy.

is observed in the galaxy, it is not near the SN location, and in any event shows no obvious indication of being associated with SF activity.

\subsection{HST Photometry}

Post-explosion $H S T$ data exist for the location of SN $2005 \mathrm{cz}$ (taken on 2006 November 11 with the Advanced Camera for Surveys/Wide Field Camera (ACS/WFC) F435W, F555W, and F814W filters and exposure times of 1500, 1500, and $1600 \mathrm{~s}$, respectively). These observations were made as part of $H S T$ program GO 10498 (PI: S. Smartt). Photometric analysis of the objects close to the location of SN $2005 \mathrm{cz}$ has been performed using point-spread function fitting procedures within IRAF DAOPHOT.

Figure 3 shows a section of the post-explosion ACS F435W image. The plotted circle has $1^{\prime \prime}$ radius $(\sim 140 \mathrm{pc})$ and is centered on the reported position of SN $2005 \mathrm{cz}$. As can be seen, we found no object suggesting the existence of massive stars nearby the reported location of $\mathrm{SN} 2005 \mathrm{cz}$. The closest source we observe is $\sim 1^{\prime \prime} .5$ ( $\sim 210$ pc, projected; see Figure 3 ); the $V-I$ colors $(\sim 1.0)$ and absolute magnitudes (ranging between -7 and -9 mag) of objects within $6^{\prime \prime}(\sim 850 \mathrm{pc})$ are all consistent with those of globular clusters, as seen in other nearby elliptical galaxies (e.g., M87; Kundu et al. 1999).

One of the scenarios discussed by Kawabata et al. (2010) suggested the progenitor of SN $2005 \mathrm{cz}$ had been a massive star $\left(10-12 M_{\odot}\right)$ with a binary companion. Massive stars usually have similarly high-mass companions (e.g., Kobulnicky \& Fryer 2007). We therefore initially attempted to find a detection of the (likely) massive companion of the $\mathrm{SN}$ progenitor in the post-explosion data, but no point source was visible at the SN site. We therefore derived detection limits for each image, in an attempt to constrain the luminosity, and hence mass, of the unseen companion of the progenitor star, or alternatively, of any other massive star likely to exist in this region if it were an SFR environment. Photon counts at the $5 \sigma$ level were defined for an aperture of 4 (ACS/WFC) pixels radius. Aperture corrections (from a 4 pixel to infinite aperture) were taken 


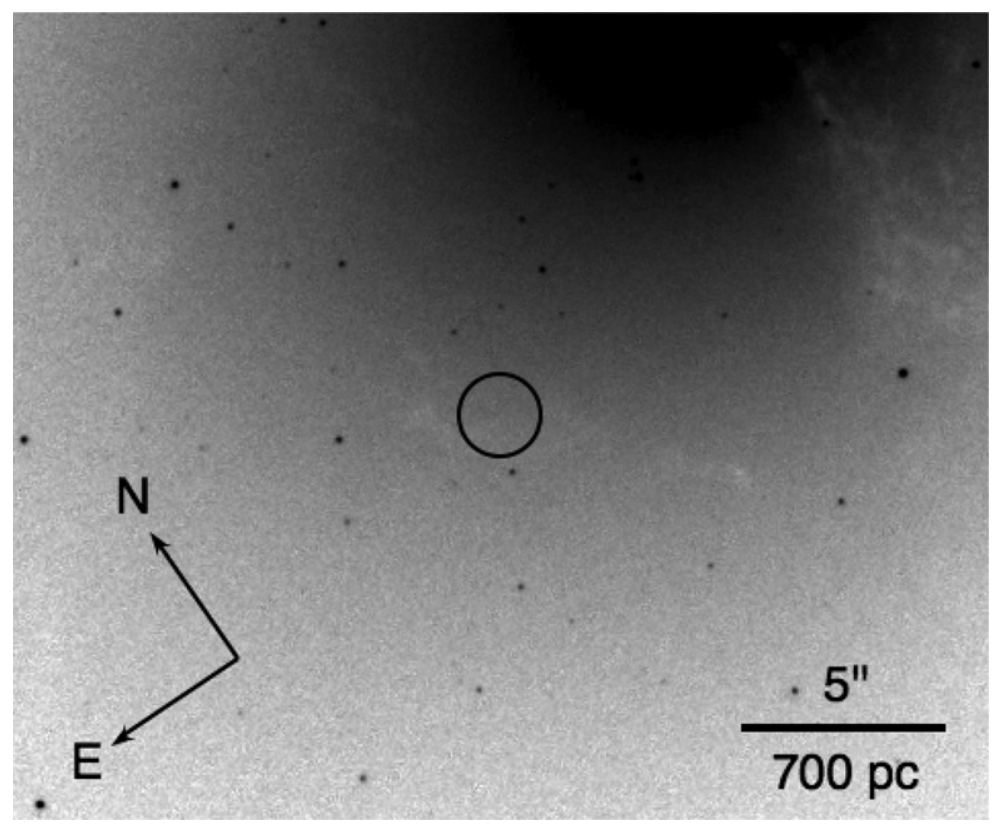

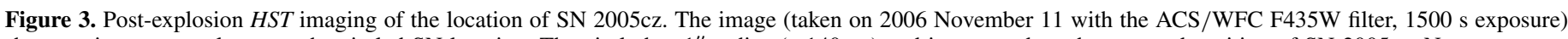

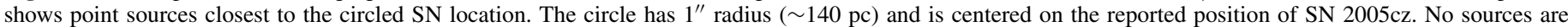
observed within this region. The observed point sources further away are consistent with being globular clusters (see the text).

from Sirianni et al. (2005), while Vegamag zero points were taken from the ACS Web site hosted by STScI. ${ }^{9}$ The apparent magnitude limits were converted to absolute magnitude limits using the average distance modulus from NED: $m-M=$ $32.27 \pm 0.62 \mathrm{mag}$ and the foreground extinction from Schlegel et al. (1998): $E(B-V)=0.028$ assuming the reddening law of Cardelli et al. (1989). We then find $5 \sigma$ detection limits of $M(\mathrm{~F} 435 \mathrm{~W})=-6.24 \pm 0.62, M(\mathrm{~F} 555 \mathrm{~W})=-6.85 \pm 0.62$, and $M(\mathrm{~F} 814 \mathrm{~W})=-7.95 \pm 0.62$, where the errors are dominated by the uncertainty in the distance modulus.

We similarly use pre-explosion HST data from the WFPC2 instrument which exist for the location of SN 2005cz. In principle, the pre-explosion data can be used to constrain not only the type of the companion of the progenitor star (or other massive stars in its environment), but also the SN progenitor itself. Unfortunately, the pre-explosion data are of significantly lower depth than post-explosion observations. These data were taken on 1994 May 14 (GO 5454; PI: M. Franx) with the F555W and F814W filters and exposure times of 1000 and 460 s, respectively, and on 1999 January 10 (SNAP 6357; PI: W. Jaffe) with the F702W filter and an exposure time of $1000 \mathrm{~s}$. We derived detection limits for these images using the same procedure as above; $5 \sigma$ photon counts defined for a 4 (PC) pixel radius aperture, with aperture corrections from Holtzman et al. (1995), and the updated Vegamag zero points of Dolphin (2000) taken from his Web site. ${ }^{10}$ In this case, we find detection limits of $M(\mathrm{~F} 555 \mathrm{~W})=-7.70 \pm 0.62, M(\mathrm{~F} 702 \mathrm{~W})=-7.77 \pm 0.62$, and $M(\mathrm{~F} 814 \mathrm{~W})=-9.32 \pm 0.62$, where again the errors are dominated by the uncertainty in the distance modulus.

The absolute magnitude limits we find are converted to luminosity limits for a range of Wolf-Rayet (W-R) and supergiant spectral types. To do so requires a color and bolometric correction for each spectral type in each of the $H S T$ instrument and filter combinations. This is exactly the same approach used by, for example, Maund \& Smartt (2005) and Crockett et al.

\footnotetext{
9 http://www.stsci.edu/hst/acs/analysis/zeropoints

$10 \mathrm{http}: / /$ purcell.as.arizona.edu/wfpc2_calib
}

(2007). Here, we have used the color and bolometric corrections for O9 to M5 supergiant stars from Drilling \& Landolt (2000). The color transformations between the ACS Vegamag and Johnson-Cousins photometry were carried out using the methodology and coefficients from Sirianni et al. (2005) for the post-explosion data. For the pre-explosion data correction from the HST flight system to Johnson-Cousins magnitudes was done following Dolphin (2000) and using the updated coefficients from his Web site. ${ }^{10}$ The results are shown in Figure 4. The solid, black curves to the right of the H-R diagrams (HRDs), which are labeled $B, V, R$, and $I$, denote the luminosity limits derived from the F435W, F555W, F702W, and F814W images, respectively. The dashed curves are the $1 \sigma$ uncertainties of these limits.

For W-R stars we have taken a simplified approach, following Crockett et al. (2007). In this case, the F555W and F435W filters provide the most restrictive limits for such hot stars in the pre- and post-explosion data (respectively), and these are the only limits that are plotted on the HRDs, shown as horizontal black lines in the top left. These lines denote the W-R luminosity limits calculated from the F555W and F435W absolute magnitude limits, and using a bolometric correction of -4.5 (Smith \& Maeder 1989). The lower, horizontal dashed lines mark the luminosity limits assuming a lower limit to the bolometric correction of -2.7 (Crowther 2007) and the lower bound of the distance modulus.

Also plotted on the HRDs are stellar evolutionary models created using the Cambridge STARS code (Eldridge \& Tout 2004). These models are of solar metallicity $(z=0.02)$ and range in mass from $10 M_{\odot}$ to $200 M_{\odot}$. W-R mass-loss rates including scaling with metallicity are from Eldridge \& Vink (2006).

The HST images show no evidence of red supergiants more massive than $\sim 15 M_{\odot}$ in the vicinity of the SN location. If the progenitor of SN $2005 \mathrm{cz}$ was a $10-12 M_{\odot}$ star (lifetimes of 18-26 Myr; Schaller et al. 1992), we might still have been able to find more massive stars (e.g., $15 M_{\odot}$, with lifetime of $\sim 13$ Myr), given a few Myr age spread inferred for SF regions (e.g., Da Rio et al. 2010; Jeffries 2009, and references therein). 
We conclude that the HST data constrain the possibility of recent SF activity, and disfavor a very massive stellar progenitor for SN $2005 \mathrm{cz}$ (i.e., likely excluding the possibility of a single massive progenitor, as suggested in some models for $\mathrm{SN} \mathrm{Ib).}$ They also exclude the existence of a massive $\left(\simeq 15 M_{\odot}\right)$ red supergiant binary companion to the progenitor of SN $2005 \mathrm{cz}$. However, these data cannot, by themselves, strongly constrain a core-collapse model with a $10-12 M_{\odot}$ progenitor for the SN.

\subsection{GALEX, 2MASS and RCS Photometry}

\subsubsection{Star Formation History of the Host Galaxy}

We use host galaxy photometry compiled in a similar way to that described by Neill et al. (2009), including ultraviolet data from the Galaxy Evolution Explorer (GALEX) satellite, and optical photometry from the third reference catalog of bright galaxies (RC3; Corwin et al. 1994) and Two Micron All Sky Survey (2MASS; Skrutskie et al. 2006). We analyzed the data in order to estimate the SF history and age of NGC 4589 using the methods described in detail by Sullivan et al. (2006, 2010). This makes use of the photometric redshift code Z-PEG (Le Borgne \& Rocca-Volmerange 2002) based upon the PEGASE.2 spectral synthesis code (e.g., Fioc \& Rocca-Volmerange 1997). Our best-fitted spectrum is shown in Figure 5.

We find that the photometry of NGC 4589 is best fitted with a total stellar mass of $10^{11.17} M_{\odot}$ (acceptable solutions are in the range $10^{11.15}-10^{11.19} M_{\odot}$ ), null specific SF rate, and a mean age of $12.5 \mathrm{Gyr},{ }^{11}$ i.e., we find no trace of any recent SF activity in this galaxy, and generally infer from the data an old age for the stellar population of NGC 4589.

\subsubsection{Limits on Local Star Formation from GALEX UV Photometry}

We have estimated a limit on the SF rate near the SN location using the measured NUV luminosity. We have relied on the relation from Kennicutt (1998) converted to the Kroupa (2001) initial mass function:

$$
\mathrm{SFR}=1.0 \times 10^{-28} \mathrm{~L}_{v, \mathrm{NUV}}
$$

where $L_{v \text { NUV }}$ is the NUV luminosity in units of erg s${ }^{-1} \mathrm{~Hz}^{-1}$ and SFR is the SF rate in $M_{\odot} \mathrm{yr}^{-1}$.

We note that SFRs derived from the NUV luminosity for red sequence galaxies are problematic (see discussion in Wyder et al. 2007), because the NUV band can include light from older stellar populations (Yi et al. 2005; Rich et al. 2005). In such cases, the NUV luminosity will overestimate the recent SFR. The SFR we find therefore serves only as an upper limit. For an aperture of $0.5 \mathrm{kpc}$ around the SN location, we find the Galactic extinction-corrected AB NUV magnitude of $22.59 \pm 0.06 \mathrm{mag}$. At the distance of NGC 4589, this translates to $\mathrm{SFR}_{\text {limit }}=4.3 \times 10^{-4} M_{\odot} \mathrm{yr}^{-1}$. At such a low SFR, massive stars are unlikely to form. Indeed, as discussed by PflammAltenburg et al. (2007), the upper limit for the stellar clusters mass function decreases with lower SFR. The fraction of ionizing massive stars is higher in heavy star clusters than in light star clusters, due to the relation between the mass of a star cluster and its most massive star. Therefore, Pflamm-Altenburg et al. (2007, see their Figure 4) find that at SFRs as low as we find, massive stars of $\sim 10 M_{\odot}$ as required for a core-collapse $\mathrm{SN}$ rarely form, if they form at all.

\footnotetext{
11 Note that the given error ranges are only statistical, and the systematics could be larger.
}
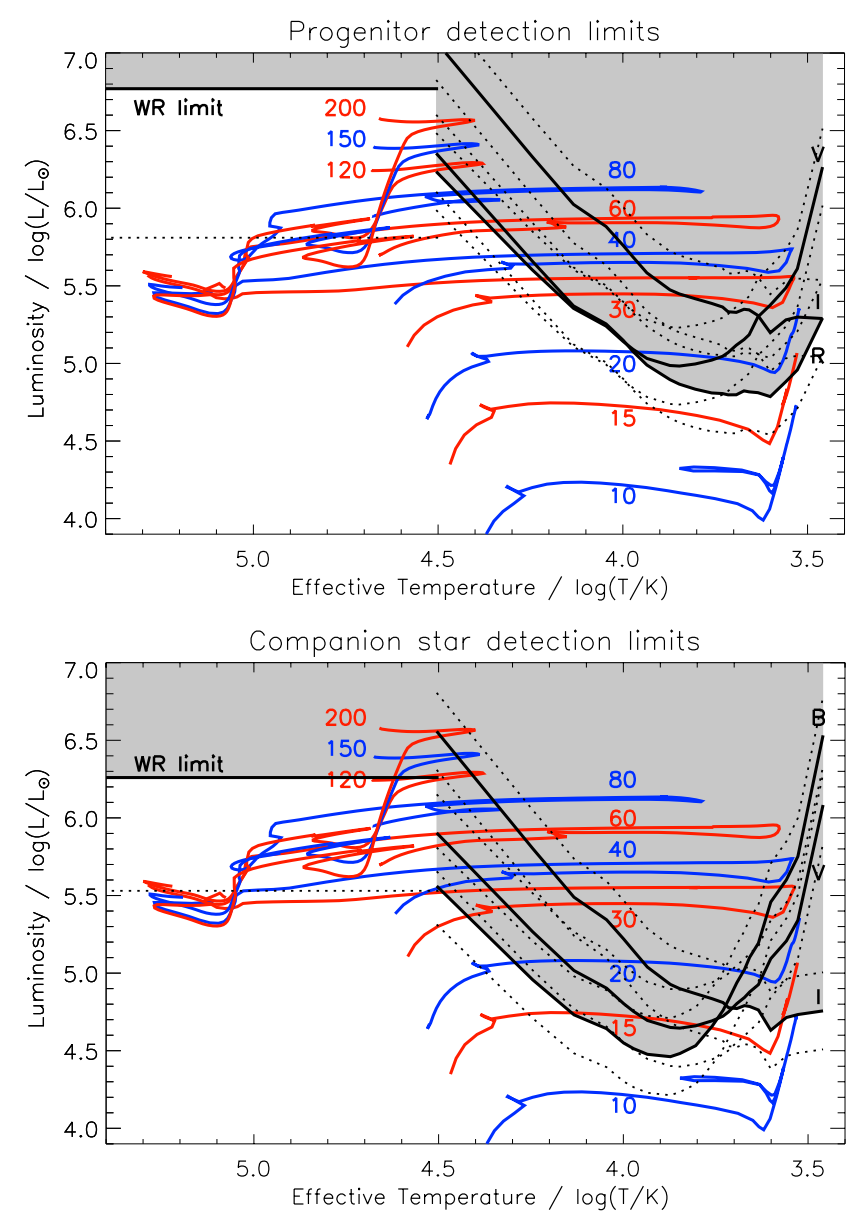

Figure 4. H-R diagrams detailing the spectral types and masses of stars that are excluded (shaded region) by the detection limits from the pre-explosion (top) and post-explosion (bottom) HST data. See the text for detailed explanations. Also plotted are stellar evolutionary models created using the Cambridge STARS code (Eldridge \& Tout 2004).

(A color version of this figure is available in the online journal.)

\subsection{Galaxy Morphology}

In support of one of the models in which SN $2005 \mathrm{cz}$ originated from a core collapse of a young massive star (10-12 $\left.M_{\odot}\right)$, Kawabata et al. (2010) suggest that NGC 4589 could have suffered a recent merger, triggering some SF activity. However, the morphology of NGC 4589 is relaxed (Schweizer et al. 1990). Even the dust lane mentioned above is observed at a very low level, and therefore a major merger occurring $100 \mathrm{Myr}$ ago or less is unlikely. Although NGC 4589 does have a complex stellar rotation field, its morphology shows a smooth optical profile following the de Vaucouleurs law (Moellenhoff \& Bender 1989); this could support the possibility that the galaxy is a relatively old merger remnant; however, this is irrelevant for the discussion of recently triggered SF. Moreover, even if a minor merger event occurred in the more recent past, the evidence from the dust lane (Figure 1) would suggest that it occurred along the minor axis, far from the SN location.

\section{DISCUSSION AND SUMMARY}

In this Letter, we studied the local and global environment of SN $2005 \mathrm{cz}$ in the elliptical galaxy NGC 4589. We used various $\mathrm{SF}$ tracers including optical spectroscopy, $\mathrm{H} \alpha$ emission, UV emission, and HST photometry. We also reviewed the merger history of the host galaxy. We found that although 


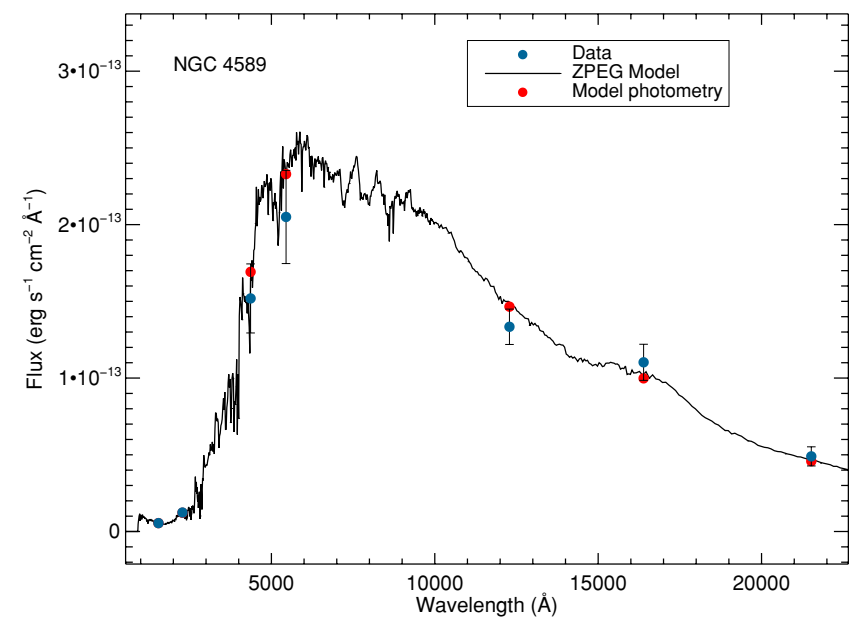

Figure 5. Spectrum of NGC 4589, fitted using the Z-PEG code. The best-fit parameters are a total stellar mass of $10^{11.17} M_{\odot}$, null specific SF rate, and mean age of $12.5 \mathrm{Gyr}$.

(A color version of this figure is available in the online journal.)

some $\mathrm{H} \alpha$ emission (which in principle could trace SF activity) exists in the host galaxy, it is far (>1.5 kpc away) from and unrelated to the close environment of SN 2005cz. Moreover, this emission is more likely to be associated with an active galactic nucleus, rather than trace SF activity. Other SF tracers (HST imaging of young massive stars, UV, $R$-band imaging, and host galaxy spectrum) show no evidence for SF in the galaxy, and particularly close to the reported location of the SN. The UV emission data could trace stars down to lower mass than $\mathrm{H} \alpha$ emission (e.g., Gogarten et al. 2009). Therefore, while $\mathrm{H} \alpha$ emission may not be detected in older SF regions, in which corecollapse $\mathrm{SNe}$ from progenitors of $10-12 M_{\odot}$ may explode, they should still present significant UV emission. The lack of such UV emission therefore suggests that recent SF activity has not occurred in this galaxy, and in particular close to the location of SN 2005cz. In addition, the overall structure and colors of the host galaxy show no evidence of recent SF in the last Gyr. The $H S T$ data exclude very massive progenitors, suggested to be the progenitors of $\mathrm{SNe} \mathrm{Ib}$, and show no evidence for massive young clusters or $>15 M_{\odot}$ supergiants near the SN location.

In principle, a massive progenitor could have formed far from the observed SN location, and later have been ejected at high velocity to explode far from its birthplace. Since we find no evidence for SF even up to $1 \mathrm{kpc}$ from the SN location, such a star should have been a runaway star to form so far (velocities of 30-100 km s${ }^{-1}$, for a lifetime of 10-40 Myr). According to Kawabata et al. (2010) the progenitor is suggested to be a binary star. However, runaway (or hypervelocity) binary stars, especially massive ones as required for an $\mathrm{SN}$ progenitor, are rare (Leonard \& Duncan 1990; Perets 2009; Perets et al. 2010; H. B. Perets \& L. Subr 2011, in preparation). Therefore, although possible in principle, such a scenario would require fine tuned conditions.

Taken together, the analysis of UV and $\mathrm{H} \alpha$ emission, the spectrum of the host galaxy NGC 4589, the 2MASS and RC3 photometry, as well as our HST data show no evidence for recent SF near the location of SN $2005 \mathrm{cz}$ or even at large distances from it.

We conclude that our results strongly disfavor a young massive-star progenitor for SN 2005cz. These results are consistent with the host galaxy type of other Ca-rich faint $\mathrm{SNe}$ $\mathrm{Ib}$, found to be biased toward early-type galaxies (Perets et al. 2010). Moreover, the only $\mathrm{SNe}$ of types II or $\mathrm{Ib} / \mathrm{c}$ ever to be found in elliptical galaxies (SNe 2000ds and 2005cz) are both faint Ca-rich SNe Ib similar to SN 2005E (Hakobyan et al. 2008; Perets et al. 2010). This provides additional support to the suggested origin of these $\mathrm{SNe}$ from a helium detonation in a WD-WD binary system, i.e., from a low-mass old progenitor rather than the core collapse of a young massive star.

We gratefully acknowledge NASA, the Centre National d'Etudes Spatiales of France, and the Korean Ministry of Science and Technology for the development, construction, operation, and science analysis of the GALEX (Galaxy Evolution Explorer) mission.

\section{REFERENCES}

Anderson, J. P., \& James, P. A. 2009, MNRAS, 399, 559

Cardelli, J. A., Clayton, G. C., \& Mathis, J. S. 1989, ApJ, 345, 245

Chu, Y.-H., \& Gruendl, R. A. 2008, in ASP Conf. Ser. 387, Massive Star Formation: Observations Confront Theory, ed. H. Beuther, H. Linz, \& T. Henning (San Francisco, CA: ASP), 415

Corwin, H. G., Jr., Buta, R. J., \& de Vaucouleurs, G. 1994, AJ, 108, 2128

Crockett, R. M., et al. 2007, MNRAS, 381, 835

Crowther, P. A. 2007, ARA\&A, 45, 177

Da Rio, N., Gouliermis, D. A., \& Gennaro, M. 2010, ApJ, 723, 166

Dimai, A., Sehgal, A., Newton, J., Puckett, T., Itagaki, K., Nakano, S., \& George, D. 2005, IAU Circ., 8569, 1

Dolphin, A. E. 2000, PASP, 112, 1397

Drilling, J. S., \& Landolt, A. U. 2000, in Normal Stars: Allen's Astrophysical Quantities, ed. A. N. Cox (4th ed.; New York: AIP), 381

Eldridge, J. J., \& Tout, C. A. 2004, MNRAS, 348, 201

Eldridge, J. J., \& Vink, J. S. 2006, A\&A, 452, 295

Filippenko, A. V. 1997, ARA\&A, 35, 309

Fioc, M., \& Rocca-Volmerange, B. 1997, A\&A, 326, 950

Gogarten, S. M., et al. 2009, ApJ, 691, 115

Goudfrooij, P., Hansen, L., Jorgensen, H. E., \& Norgaard-Nielsen, H. U. 1994, A\&AS, 105, 341

Hakobyan, A. A., Petrosian, A. R., McLean, B., Kunth, D., Allen, R. J., Turatto, M., \& Barbon, R. 2008, A\&A, 488, 523

Ho, L. C., Filippenko, A. V., \& Sargent, W. L. W. 1997, ApJS, 112, 315

Holtzman, J. A., Burrows, C. J., Casertano, S., Hester, J. J., Trauger, J. T., Watson, A. M., \& Worthey, G. 1995, PASP, 107, 1065

Jeffries, R. D. 2009, IAU Symp. 258, The Ages of Stars, ed. E. E. Mamajek, D. R. Soderblom, \& R. F. G. Wyse (Cambridge: Cambridge Univ. Press), 95 Kawabata, K. S., et al. 2010, Nature, 465, 326

Kennicutt, R. C., Jr. 1998, ARA\&A, 36, 189

Kobulnicky, H. A., \& Fryer, C. L. 2007, ApJ, 670, 747

Kroupa, P. 2001, MNRAS, 322, 231

Kundu, A., et al. 1999, ApJ, 513, 733

Le Borgne, D., \& Rocca-Volmerange, B. 2002, A\&A, 386, 446

Leonard, P. J. T., \& Duncan, M. J. 1990, AJ, 99, 608

Maund, J. R., \& Smartt, S. J. 2005, MNRAS, 360, 288

Moellenhoff, C., \& Bender, R. 1989, A\&A, 214, 61

Neill, J. D., et al. 2009, ApJ, 707, 1449

Perets, H. B. 2009, ApJ, 698, 1330

Perets, H. B., et al. 2010, Nature, 465, 322

Pflamm-Altenburg, J., Weidner, C., \& Kroupa, P. 2007, ApJ, 671, 1550

Rich, R. M., et al. 2005, ApJ, 619, L107

Sandage, A., \& Visvanathan, N. 1978, ApJ, 225, 742

Schaller, G., Schaerer, D., Meynet, G., \& Maeder, A. 1992, A\&AS, 96, 269

Schilbach, E., \& Röser, S. 2008, A\&A, 489, 105

Schlegel, D. J., Finkbeiner, D. P., \& Davis, M. 1998, ApJ, 500, 525

Schweizer, F., Seitzer, P., Faber, S. M., Burstein, D., Dalle Ore, C. M., \& Gonzalez, J. J. 1990, ApJ, 364, L33

Shen, K. J., Kasen, D., Weinberg, N. N., Bildsten, L., \& Scannapieco, E. 2010, ApJ, 715, 767

Sirianni, M., et al. 2005, PASP, 117, 1049

Skrutskie, M. F., et al. 2006, AJ, 131, 1163

Smith, L. F., \& Maeder, A. 1989, A\&A, 211, 71

Sullivan, M., et al. 2006, ApJ, 648, 868

Sullivan, M., et al. 2010, MNRAS, 406, 782

Waldman, R., Sauer, D., Livne, E., Perets, H., Glasner, A., Mazzali, P., Truran, J. W., \& Gal-Yam, A. 2010, arXiv:1009.3829

Wyder, T. K., et al. 2007, ApJS, 173, 293

Yi, S. K., et al. 2005, ApJ, 619, L111 